\title{
The fusiform gyrus exhibits an epigenetic signature for Alzheimer's disease
}

Dingailu Ma ${ }^{1,2,3}$, Irfete S. Fetahu ${ }^{3}$, Mei Wang ${ }^{4}$, Rui Fang ${ }^{3}$, Jiahui Li ${ }^{1,2}$, Hang Liu ${ }^{1,2}$, Tobin Gramyk ${ }^{3}$, Isabella Iwanicki ${ }^{3}$, Sophie $\mathrm{Gu}^{3}$, Winnie $\mathrm{Xu^{1,2 }}$, Li Tan ${ }^{1,2}$, Feizhen $\mathrm{Wu}^{1,2^{*}}$ and Yujiang G. Shi ${ }^{3^{*}}$ (I)

\begin{abstract}
Background: Alzheimer's disease (AD) is the most common type of dementia, and patients with advanced AD frequently lose the ability to identify family members. The fusiform gyrus (FUS) of the brain is critical in facial recognition. However, AD etiology in the FUS of AD patients is poorly understood. New analytical strategies are needed to reveal the genetic and epigenetic basis of AD in FUS.
\end{abstract}

Results: A complex of new analytical paradigms that integrates an array of transcriptomes and methylomes of normal controls, AD patients, and "AD-in-dish" models were used to identify genetic and epigenetic signatures of AD in FUS. Here we identified changes in gene expression that are specific to the FUS in brains of AD patients. These changes are closely linked to key genes in the $A D$ network. Profiling of the methylome $(5 \mathrm{mC} / 5 \mathrm{hmC} / 5 \mathrm{fC} /$ $5 \mathrm{caC}$ ) at base resolution identified 5 signature genes (COL2A1, CAPN3, COL14A1, STAT5A, SPOCK3) that exhibit perturbed expression, specifically in the FUS and display altered DNA methylome profiles that are common across AD-associated brain regions. Moreover, we demonstrate proof-of-principle that AD-associated methylome changes in these genes effectively predict the disease prognosis with enhanced sensitivity compared to presently used clinical criteria.

Conclusions: This study identified a set of previously unexplored FUS-specific AD genes and their epigenetic characteristics, which may provide new insights into the molecular pathology of $A D$, attributing the genetic and epigenetic basis of FUS to AD development.

Keywords: Alzheimer's disease, Fusiform gyrus, Base-resolution DNA methylome analysis, Genome-wide transcriptome analysis, Protein-protein interaction networks, Postmortem brain, iPSC-derived neuron, Epigenetics

\section{Background}

Alzheimer's disease (AD) is a progressive neurodegenerative disorder with no cure or reliable methods for early detection [1-4]. Genetic studies show that earlyonset $\mathrm{AD}$ (EOAD) (< 65 years old), accounting for only $5 \%$ of the $\mathrm{AD}$ population, is associated with

\footnotetext{
*Correspondence: wufz@fudan.edu.cn; yujiang_shi@hms.harvard.edu 'Laboratory of Epigenetics, Institutes of Biomedical Sciences, Fudan University, Shanghai 200032, China

${ }^{3}$ Division of Endocrinology, Diabetes and Hypertension, Department of Medicine, Brigham and Women's Hospital, Harvard Medical School, Boston, MA 02115, USA

Full list of author information is available at the end of the article
}

mutations in the APP, PSEN1, and PSEN2 genes. Meanwhile, 50\% of late-onset AD (LOAD) cases are attributed to homozygous APOE4 [5]. However, the majority of $\mathrm{AD}$ cases are sporadic and cannot be explained by genetic variations, suggesting the existence of yet unknown etiology [5].

The symptoms and severity of $\mathrm{AD}$ vary in patients [6-8], which may be associated with the affected brain areas and lesion invasion rate. The fusiform gyrus (FUS) is a structure that lies on the basal surface of the temporal and occipital lobes in Brodmann's area 37. It contains the critical fusiform face area (FFA) responsible for facial recognition [9]. Patients with advanced AD frequently lose 
the ability to identify family members. Likewise, subjects with mild cognitive impairment (MCI), who experience a higher risk of conversion to $\mathrm{AD}$, possess distinct changes in functional connectivity of the FUS [10]. Therefore, ADlinked genes in the FUS may be critical in AD onset and progression and are thus promising targets for early diagnosis and therapy. However, compared with well-studied and documented brain areas such as the hippocampus (HPC) [11], prefrontal cortex (PFC) [12], and temporal lobe (TPL) [13], the gene expression characteristics and molecular mechanisms of action involved in AD pathology in the FUS [14] remain unexplored. The identification of AD-related expression and epigenetic signatures in different brain regions can provide support of inherent molecular mechanisms for the heterogeneous symptoms and improve the individualized early detection, prevention, and treatment of AD patients [15].

Growing lines of evidence suggest that epigenetic mechanisms play a crucial role in AD onset and development $[13,16,17]$. DNA methylation at the 5th position of cytosine $(5 \mathrm{mC})$ can be oxidized into 5hydroxymethylcytosine $(5 \mathrm{hmC}), 5$-formylcytosine $(5 \mathrm{fC})$, and 5-carboxylcytosine $(5 \mathrm{caC})$ (hereafter referred to as $5 \mathrm{mC}, 5 \mathrm{hmC}, 5 \mathrm{fC}$, and $5 \mathrm{caC}$ ) by the family of ten-eleven translocation enzymes (TET1/TET2/TET3) in a stepwise manner [18]. Previous studies have indicated that AD onset and progression are linked to specific changes in DNA methylation in affected brain regions [19-21]. Our recent genome-wide DNA methylome analysis of postmortem brains and iPSC-derived neurons at base resolution identified a roadmap of $\mathrm{AD}$-specific epigenetic signatures [17]. Moreover, the DNA methylome can be changed before the accumulation of pathological lesions and clinical manifestation [22-27]. In-depth study of mechanisms of gene expression and DNA methylation regulation in different brain regions of $A D$ have shed new light on molecular markers of AD. Once verified in future, these specific sets of markers will have valuable implications for the early diagnosis of AD.

In this study, using a new analytical strategy, we uncovered an unprecedented FUS-specific AD gene expression profile and described an epigenetic basis for how $\mathrm{AD}$-related changes extend to other brain regions beyond the FUS. Using independent methylation datasets from AD patients, we identified 5 genes (COL2A1, CAPN3, COL14A1, STAT5A, and SPOCK3) with a methylome signature that was significantly associated with AD prognosis.

\section{Results}

\section{Changes in AD-specific gene networks are linked to specific brain regions}

By comparing transcriptomes of AD patients with normal controls $(n=108)$ in 4 different brain regions, we identified 2861 differentially expressed (DE) genes in the fusiform gyrus (FUS), 716 in the hippocampus (HPC), 375 in the prefrontal cortex (PFC), and 2166 in the temporal lobe (TPL) (Fig. 1a, Table S1). The top 3 enriched Kyoto Encyclopedia of Genes and Genomes (KEGG) pathways associated with the DE genes were distinct across brain regions, while sharing some similarities (Fig. 1a). DE genes in the HPC, the first affected area during $\mathrm{AD}$ onset, were enriched in cytokine-cytokine receptor interactions and the IL-17 signaling pathway, which are involved in acute and chronic inflammatory responses. In contrast, in the FUS, which is presumed to be a lateraffected area in $\mathrm{AD}$ progression, the top-enriched pathways were neuroactive ligand-receptor interaction and the cAMP signaling pathway. Furthermore, DE gene overlap analysis among the four brain regions revealed that very few genes were consistently differentially expressed in more than two brain regions, and most genes were specifically affected in one unique brain region (Fig. 1b). We should note that comparing the $\mathrm{DE}$ analysis between brain regions only in controls, we found 4315 overlapping DE genes were differentially expressed in FUS, while there were 865, 1993, and 1512 overlapping DE genes in HPC, TPL, and PFC, respectively (Figure S1a-d). The apparent difference between DE genes among brain regions only in control and in AD samples suggests the unique gene expression regulation pattern in the context of AD.

Some of the KEGG pathways were significantly enriched in more than one brain region. We found that DE genes in both the FUS and TPL were significantly associated with neuroactive ligand-receptor interactions, while the HPC and PFC DE genes were associated with cytokine-cytokine receptor interaction. In addition, DE genes in the FUS and HPC were closely associated with the PI3K-Akt signaling pathway. Intriguingly, although DE genes in both the FUS and HPC were enriched in the PI3K/AKT/GSK-3 $\beta$ pathway, which is likely activated by neurotrophins and plays critical roles in $\mathrm{AD}$ onset and progression $[28,29], 84 \%$ (21 of 25 ) of the DE genes associated with this pathway in the FUS were upregulated in AD patients compared to controls (Fig. 1c). In contrast, the majority of these genes were downregulated or unchanged in the HPC. These gene expression patterns were also found in pathways related to neuroactive ligand-receptor interactions and cytokine-cytokine receptor interactions (Figure S1e-f). For example, we observed downregulation of GABA receptor genes in the FUS and TPL of AD brains (labeled with an arrow in Figure S1a). Interestingly, it was recently reported that secreted APP may modulate synaptic 


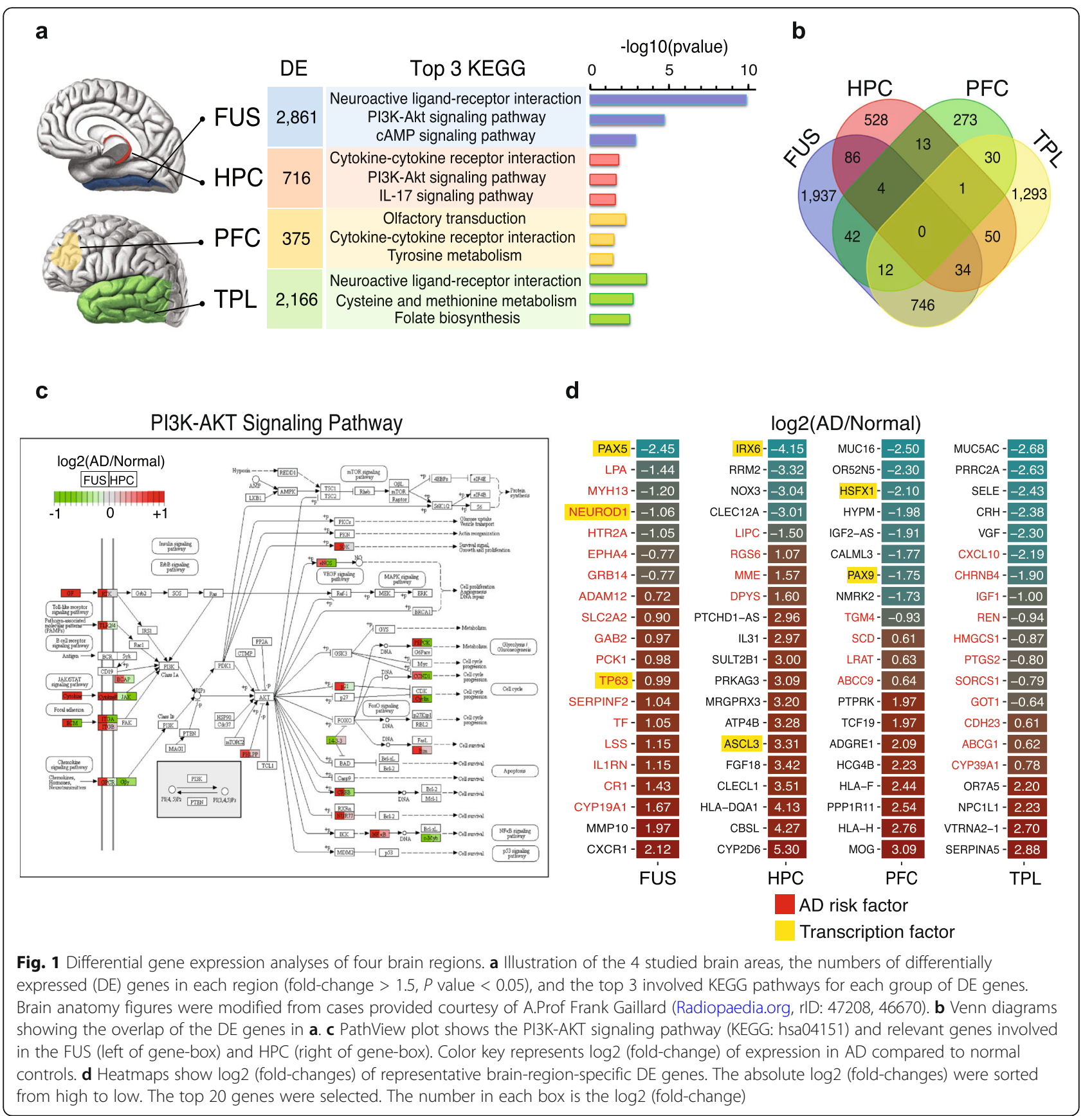

transmission via GABA receptors. Downregulation of the GABA receptor gene in the FUS and TPL is possibly linked to aberrant synaptic transmission in ADaffected neurons [30]. Together, these findings strongly suggest that the gene expression pattern in these specific cellular pathways, while similarly affected, may act differently, or perhaps even in opposite, in different brain regions during $\mathrm{AD}$ progression.

Critically, through AD-linkage analysis of the top 20 DE genes specific to each region, identified based on an absolute $\log 2$ (fold-change) (Fig. 1d), we found that $45 \%$
$(36 / 80)$ of these DE genes have been previously reported as $\mathrm{AD}$ risk factors (Table S2). The definition of the list of AD risk factors was composed by taking into account the genetic testing or variation resources. Of these $\mathrm{DE}$ genes, $17 / 20$ were associated with the FUS, $4 / 20$ were associated with the HPC and PFC, and 11/20 were associated with the TPL. In addition, in order to ensure that the genes did appear in the AD GWAS results, we carefully compared the $\mathrm{AD}$ genetic datasets of the International Genomics of Alzheimer's Project (IGAP) [31] and ALZGENE [32]. 47.5\% (38/80) of the top 20 DEGs 
were identified in AD GWAS results (Table S2). We also performed the gene enrichment analysis using the hypergeometric distribution for MalaCards and IGAP, respectively, resulting in significant $P$ values $\left(P_{\text {MalaCards }}=\right.$ 0.00061, $\left.P_{\text {IGAP }}=0.03764\right)$. Taken together, these data suggest that specific genetic components are involved in different brain regions linked to $\mathrm{AD}$ onset and progression.

\section{iPSC-derived neurons recapitulate the transcription profiles of the fusiform gyrus}

We sought to further characterize possible molecular changes relating to the region-specific genetic changes found in $\mathrm{AD}$ patients. Induced pluripotent stem cells (iPSCs) derived from AD patients allowed us to accurately recapitulate neurogenesis, providing suitable models to study AD [33]. We employed four types of iPSCs: WT, PSEN1 ${ }^{\text {mut }}$, PSEN2 ${ }^{\text {mut }}$, and $\mathrm{APOE}^{\varepsilon 4 / \varepsilon 4}$, derived from normal individuals and $\mathrm{AD}$ patients carrying a PSEN1 mutation, PSEN2 mutation, and homozygous $A P O E-\varepsilon 4$, respectively. These iPSCs were then subjected to directed differentiation into neurons using a commonly available protocol [17, 34] (Fig. 2a). Unexpectedly, bioinformatic analysis showed the transcriptome profiles of WT neurons (WT-N), APOE4-N, PSEN1-N, and PSEN2-N were all strongly correlated with those of the FUS, with higher correlation coefficients than those of the HPC, PFC, and TPL (Fig. 2b). This indicated that the transcriptional features of iPSC-derived neurons were more similar to the FUS than other brain regions. PSEN2-N showed the strongest correlation with the FUS $(r=0.73, P$ value $<0.01$, Pearson's correlation), suggesting that these AD neurons, particularly PSEN2-N, may recapitulate the transcription profiles of $\mathrm{AD}$ in the FUS.

The similarity of the transcriptional profiles between the FUS and iPSC-derived cortical neurons may be due to the specific differentiation protocol used. To systematically evaluate the protocol bias-resultant similarity, we analyzed 13 up-to-date available public datasets of differentiated brain cell transcriptomes [34-48] (detailed information is summarized in Table S5) and surprisingly found that iPSC-derived cortical neurons generated following the same protocol [34] also had a stronger correlation with the FUS than other brain regions (Fig. 2d). In contrast, neurons differentiated through different protocols, such as DGgranule, forebrain, and dopamine neurons (last 6 rows in Fig. 2d), were more transcriptionally similar to the HPC, PFC, and TPL. These data again suggest that iPSC-derived cortical neurons possess similar transcriptional patterns to the FUS.
Next, we asked how many DE genes were shared between iPSC-derived AD neurons and the FUS when compared to their respective controls. Approximately $50 \%$ of DE genes in the FUS overlapped with those in iPSC-derived cortical neurons (Fig. 2c, Figure S2a-b). There were $1372 \mathrm{DE}$ genes shared between the FUS and PSEN2-N (Fig. 2c), 1388 between the FUS and PSEN1-N, and 1475 between the FUS and APOE4-N (Figure S2a-b, Table S3). We also compared the overlap of DE genes between all cell lines tested: PSEN1-N, PSEN2-N, and APOE4-N (Figure S2c). Gene ontology analysis showed that the 1372 common DE genes between the FUS and PSEN2-N were significantly enriched in extracellular matrix organization, chemical synaptic transmission, cell differentiation, and axon genesis (Figure S2d). Importantly, the top-enriched KEGG pathways in these 1372 common DE genes were also significantly enriched in the FUS DE genes (Fig. 2e). There are 292 genes that are upregulated and 262 genes that are downregulated in both the FUS and PSEN2-N (Fig. 2f, Table S4). Interestingly, these $554(292+262)$ $\mathrm{DE}$ genes were distributed in extracellular and membrane areas in the PI3K/AKT/GSK-3 $\beta$ pathway (Figure S2e), implying a relationship to signal transduction and neuronal cell survival [49]. Collectively, iPSC-derived neurons recapitulate the transcription profiles of the diseased FUS, suggesting that the molecular pathology of AD in PSEN2-N significantly mirrors that in the FUS.

\section{Protein-protein interaction networks reveal FUS-specific key AD-associated genes}

To determine if these $554 \mathrm{DE}$ genes shared by the FUS and PSEN2-N have a clear association with well-defined AD genes, we used the STRING [50] analytical tool to reveal the protein-protein interaction (PPI) networks (Fig. 3 ). Only interaction edges with a high confidence (interaction score $\geq 0.9$, PPI enrichment $P$ value $<1 \mathrm{e}-11$ ) were selected. STRING analysis identified 114 DE genes as being clustered to well-defined key AD genes in a hierarchical fashion (Table S6). For example, with the $\varepsilon 4$ allele being an $\mathrm{AD}$ risk factor [51], APOE was the primary nodule connecting to $A P P$ (red line), which encodes peptides that form amyloid plaques in AD brains (Fig. 3). The secondary nodule linked to $A P P$ is the highly expressed $L R P 2$, in which a SNP associated with AD susceptibility was found [52]. The nodule downstream of LRP2 is SH3GL2, which is implicated in synaptic vesicle endocytosis and AD protein homeostasis [53]. The last nodule is PTPN3, which is a diabetes-related gene [54]. These connections imply that LRP2, SH3GL2, and PTPN3 are possible novel AD-linked genes which play critical roles that are closely associated with the 


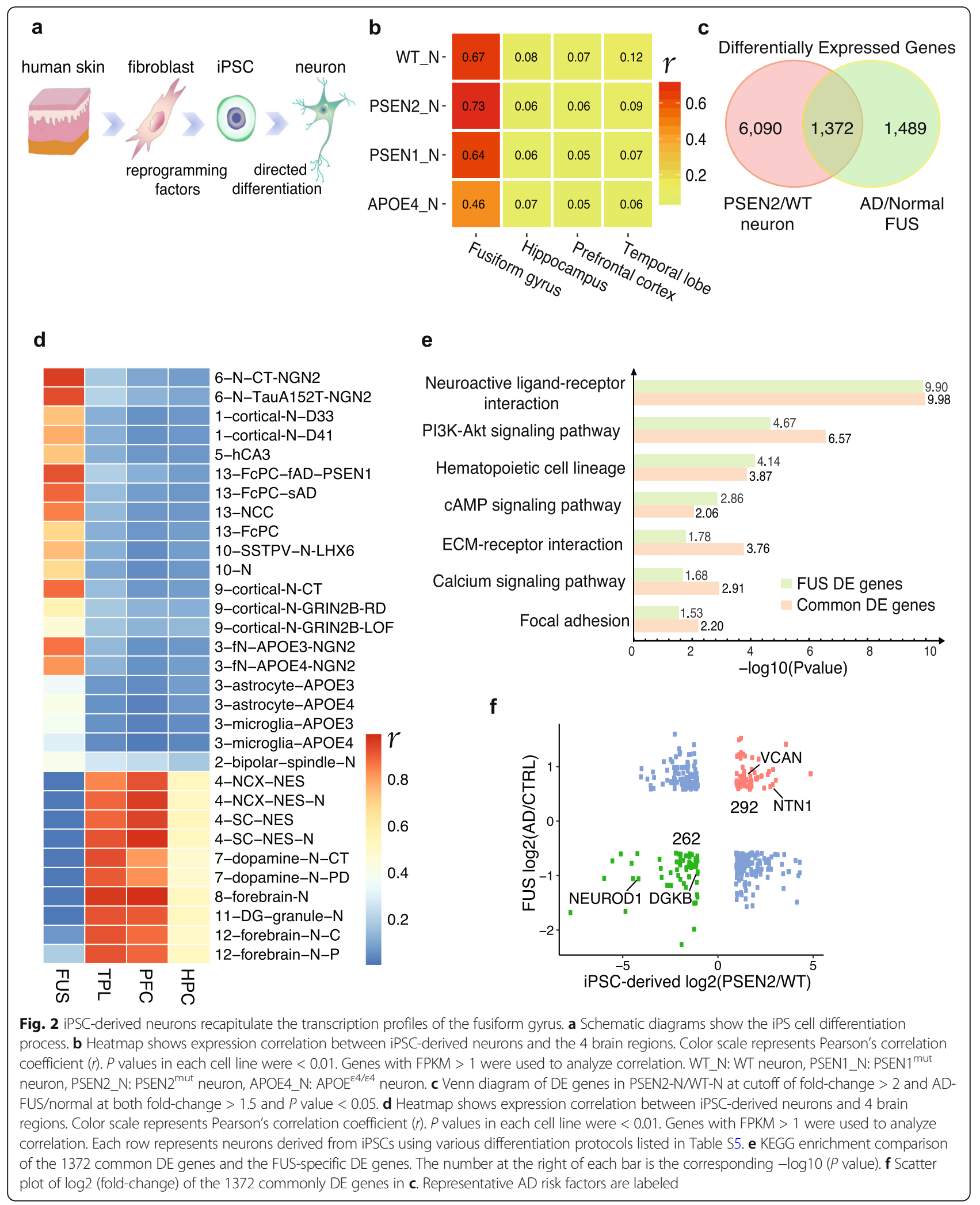

APOE4 functional network in $\mathrm{AD}$ pathology. Using the same approach, we performed PPI enrichment for PSEN1-N and APOE4-N and identified 112 and 116 enriched DE genes associated with the functional realm of the other well-defined key $\mathrm{AD}$ genes, respectively (Figure S3-4, Table S6). 


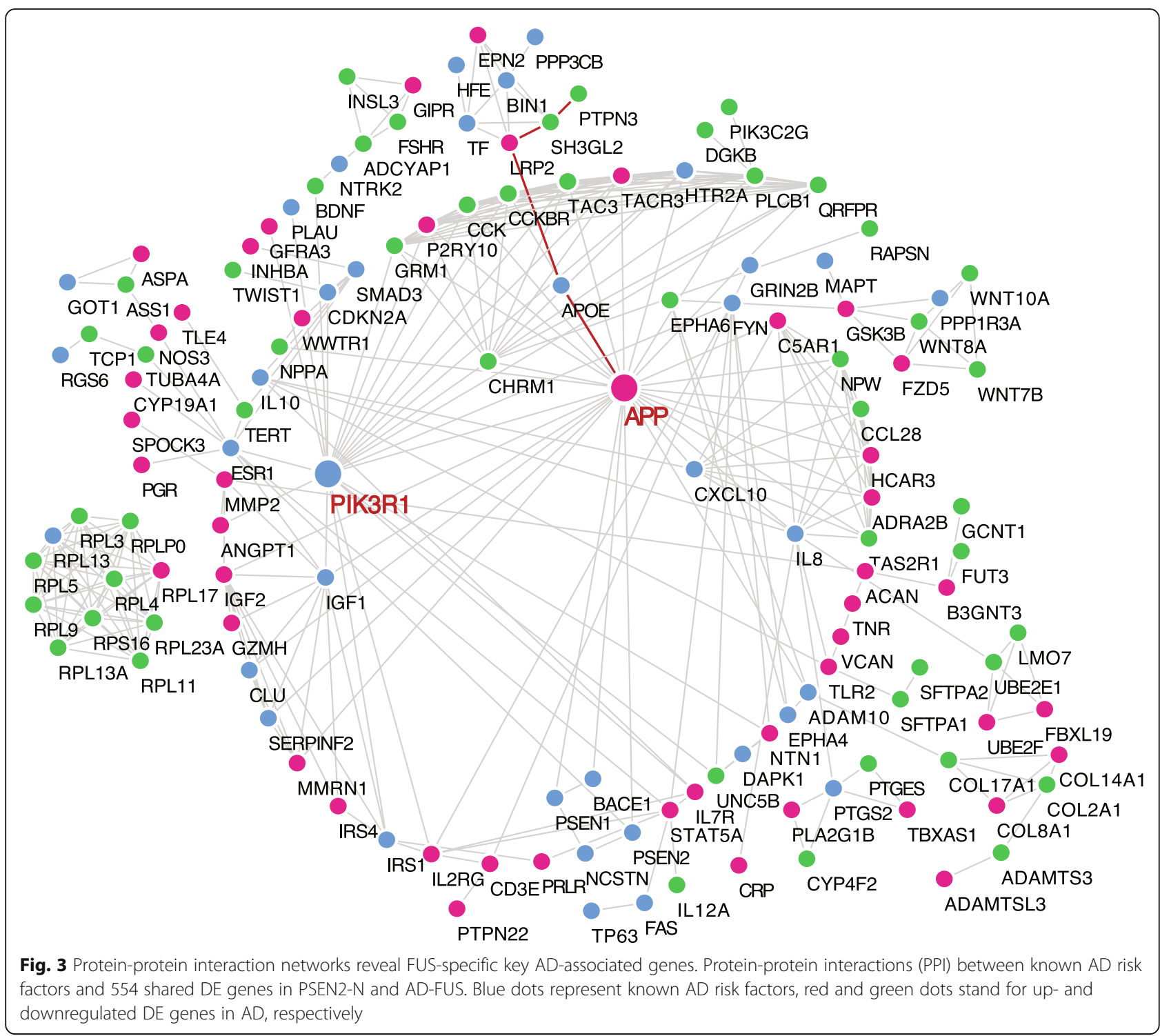

We found common and unique sub-networks among these PPI networks. The APP-centered sub-network appeared in each network (Fig. 3, Figure S3-4), indicating that $A P P$ plays a critical role in $\mathrm{AD}$ progression and that the $\mathrm{DE}$ genes associated with the APP functional nodule may be deeply involved in amyloid plaque formation. Compared to APOE4-N, PSEN1-N and PSEN2-N had a unique PIK3R1-centered sub-network (Fig. 3 and Figure S3). PIK3R1 is implicated in the metabolic actions of insulin, in which insulin receptor-activated genes play critical roles in vesicle transport and RNA splicing in neuronal cells $[55,56]$. Interestingly, the APOE4-N PPI network had distinct patterns. The $N M U$-centered subnetwork is connected to APP and was not observed in PSEN1-N and PSEN2-N (Figure S4). NMU encodes a member of the neuromedin family of neuropeptides, which play important roles in inflammation-mediated memory impairment and neuronal cell-death [57]. Taken together, these data revealed that the AD-specific gene expression signature shared by the FUS and in vitro differentiated cortical neurons is functionally linked to well-defined AD risk factors.

\section{AD-specific methylation patterns in the newly identified $A D$-specific gene expression signatures}

We identified AD-specific methylome patterns and signatures in the newly identified $\mathrm{AD}$-specific gene expression signatures (Fig. 4a). Using oxBS- and MAB-seq, we profiled whole-genome $5 \mathrm{mC}, 5 \mathrm{hmC}$, and $5 \mathrm{fC} / \mathrm{caC}$ signals at base resolution in WT-N, PSEN1-N, PSEN2-N, and APOE4-N (Figure S5a-f). The global methylation patterns in the gene bodies varied between $\mathrm{AD}$ and WT iPSC-derived neurons, suggesting that aberrant DNA methylomes may 
a

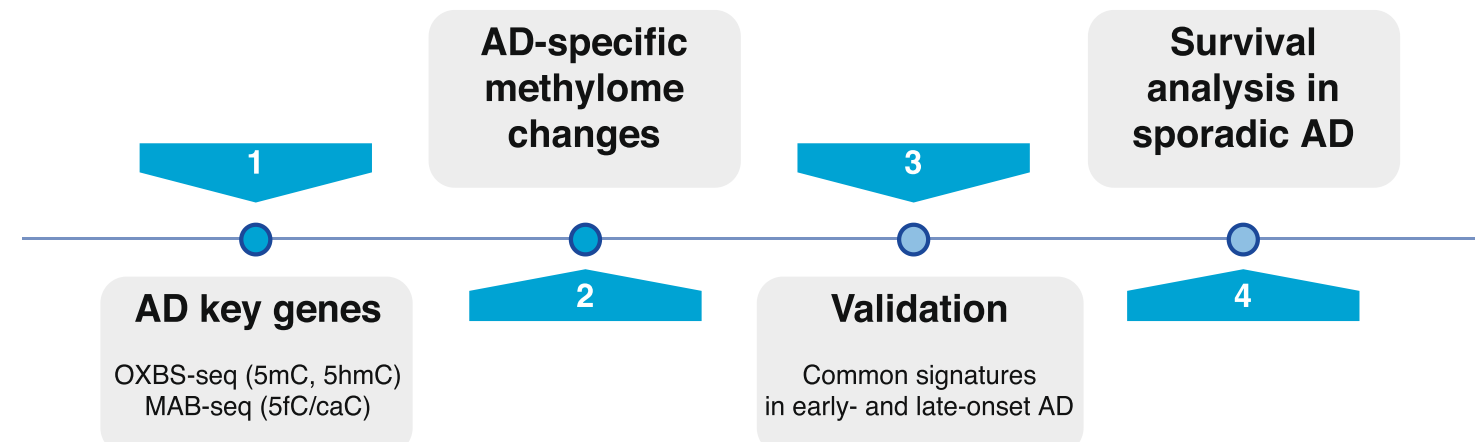

b
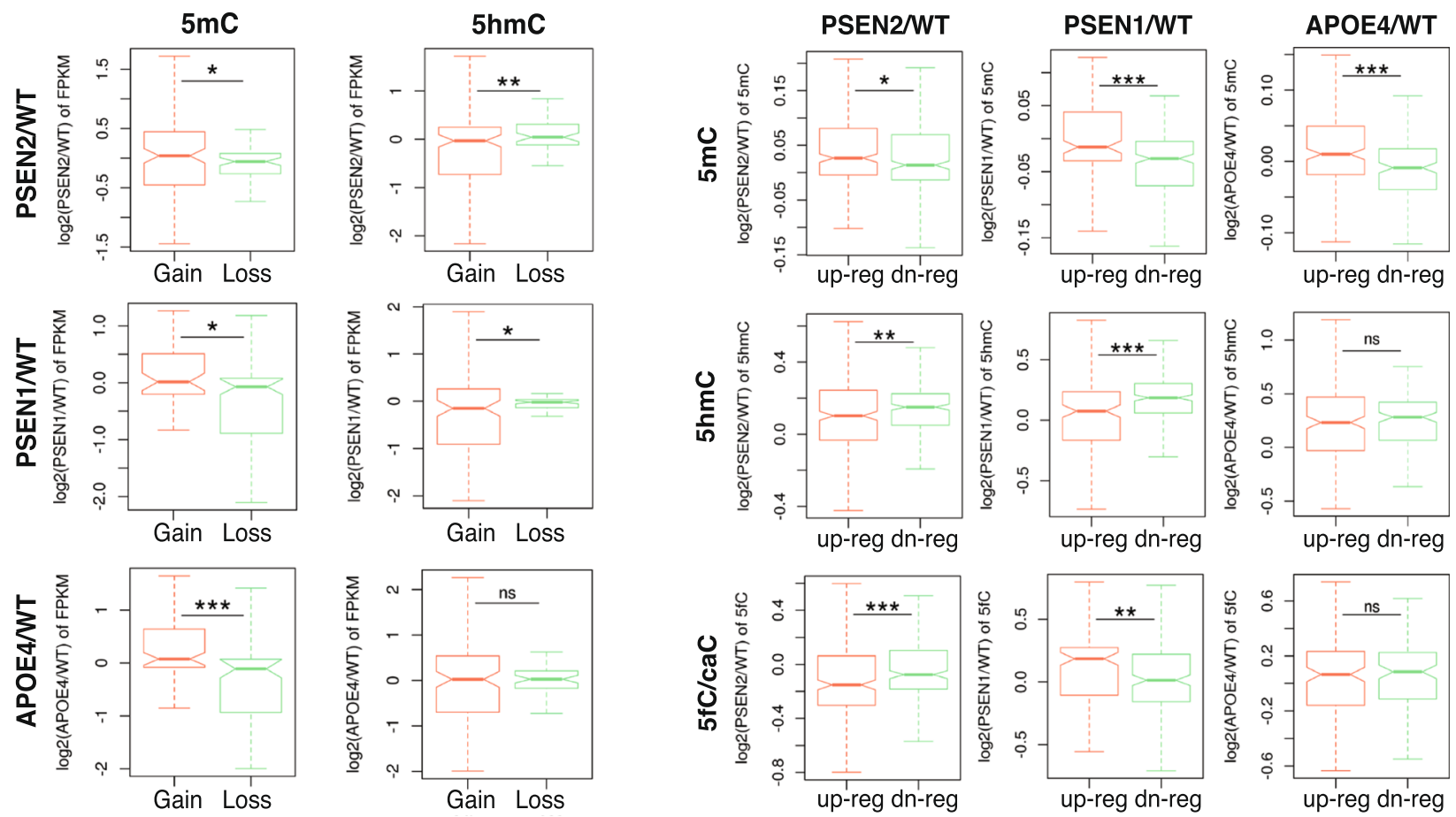

Fig. 4 AD-specific methylation patterns in the newly identified AD-specific gene expression signatures. a Schematic diagram of the analysis workflow to identify AD-specific methylome patterns and AD-methylome signatures. $\mathbf{b}$, c Boxplots show expression fold-changes in methylationgain or loss on gene bodies of PPI-enriched key AD-associated genes (b), and $5 \mathrm{mC} / 5 \mathrm{hmC} / 5 \mathrm{fC} / \mathrm{caC}$ fold-changes on the gene bodies of up- and downregulated DE genes $(\mathbf{c})$. $P$ values were calculated by the Wilcoxon rank-sum test $\left(P<0.05\left(^{*}\right)\right.$; $P<0.01\left(^{* *}\right)$; $P<0.001\left(^{* *}\right)$; ns, not significant)

contribute to transcriptional regulation in $\mathrm{AD}$ progression.

Next, we examined the correlation between the ADspecific gene expression signatures (Table S6) and the methylome. Genes were separated into two groups, gain or loss of methylation, according to the average methylation levels on their gene bodies, and then stratified by their expression fold-changes (Fig. 4b).
Compared to all Refseq genes (Figure S5g), we observed unusual perturbation of the DNA methylomes in the AD-specific expression signature genes. Consistently in PSEN2, PSEN1, and APOE4 neurons, loss of $5 \mathrm{mC}$ on the gene bodies of the signature genes correlated with lower expression in AD neurons compared to WT (Fig. 4b, left panel). On the contrary, the genes with a loss of $5 \mathrm{hmC}$ showed higher 
expression in $\mathrm{AD}$ neurons than those with a gain of $5 \mathrm{hmC}$ (Fig. 4b, right panel).

We next investigated the gene body methylation differences in the up- or downregulated signature genes (Fig. 4c). The upregulated genes tended to gain more $5 \mathrm{mC}$ on their gene bodies than the downregulated genes in PSEN2-N compared to WT-N (Fig. 4c, top panel). This trend was also found in PSEN1 and APOE4 neurons (Fig. 4c, top panel). In contrast, downregulated genes tended to gain more $5 \mathrm{hmC}$ than the upregulated genes. Collectively, AD-specific methylome changes are significantly correlated with perturbed expression of the newly identified AD-specific signature genes.

\section{Cross-validation of key AD genes in independent methylation datasets}

To validate that the identified methylation changes existed in the brain tissues of AD patients, we examined an independent cohort of 44 controls and $\mathrm{AD}$ patients and used the methylation data of the temporal cortex to verify the methylation sites we identified [58]. Our results showed that $65 / 114$ (57\%) of the newly identified signature genes in PSEN2-N had a consistent trend of methylation changes (Fig. 5a). Importantly, the topenriched pathways or Gene Ontology (GO) terms associated with these 65 validated signature genes suggested critical roles in $\mathrm{AD}$ development, including the $\mathrm{AD}$ presenilin pathway, axon guidance mediated by netrin, and nervous system development (Fig. 5b, c). Additionally, 44/112 (39\%) enriched DE genes in PSEN1-N (Figure S6a) and 54/116 (47\%) enriched DE genes in APOE4-N (Figure S6b) were validated to show a consistent trend of methylation change. Furthermore, among the validated genes, 38/65 (58\%) in PSEN2-N, 27/44 (61\%) in PSEN1-N, and 25/54 (46\%) in APOE4-N were previously reported as being closely associated with $\mathrm{AD}$ (Table S7). Among all of these validated genes, 8 genes (STAT5A, TWIST1, GBP3, GCNT1, SPOCK3, CAPN3, $C O L 14 A 1$, and COL2A1) were altered in neurons derived from patients with PSEN1, PSEN2, and APOE4 mutations (Fig. 5d, Table S7). Intriguingly, there were 9 genes (GLI1, CD3E, CRP, ANGPT1, ASS1, IGF2, INSL3, $P D E 1 A$, and PIK3C2G) that were altered only in PSEN1 and PSEN2 neurons, suggesting they might be related to EOAD-specific signatures.

Among the 8 validated common signature genes, two are transcription factors (STAT5A, TWIST1), two are associated with immune response (GBP3, GCNT1), one is a nervous system regulator ( $S P O C K 3$ ), and three are intra- or extracellular components (CAPN3, COL2A1, COL14A1) (Table S7). These genes are directly or indirectly associated with $\mathrm{AD}$ pathophysiology. For example, the JAK2-STAT5 signaling pathway plays a critical role in mediating IL-3-induced activation of microglia during
AD pathogenesis [59]. TWIST1 is targeted by an ADspecific miRNA in a set of AD patients and is an important upstream mediator of mutant $\mathrm{Htt}$ (huntingtin protein)-induced neuronal death [60]. COL14A1 is an interactive gene in the gut-brain axis in $\mathrm{AD}$ [61]. Collectively, these results indicate that the validated signature genes likely play important roles in $\mathrm{AD}$ progression and suggest that accumulation of aberrant methylomes, which may predate abnormal transcriptional changes, may function as a new set of independent epigenetic markers for early detection and prognostic evaluation of $\mathrm{AD}$.

\section{Survival analysis of validated AD signatures using the Religious Orders Study and Rush Memory and Aging Project (ROS/MAP) cohort}

To investigate whether the 8 commonly validated genes are associated with the prognosis of $\mathrm{AD}$, we evaluated the effect of changes in the methylome on these potential risk factors on the survival time of AD patients. A total of $174 \mathrm{AD}$ patients in the ROS/MAP cohort [62] were included with censored follow-up time from the first diagnosis to the age of death. Other clinical characteristics from medical records including sex, $A P O E$ genotype, BRAAK-score, and CERAD-score were included. Among the 8 genes, the methylation levels of 5 gene signatures (COL2A1, CAPN3, COL14A1, STAT5A, and $S P O C K 3)$ were detected in the ROS/MAP cohort. The Cox proportional hazards regression model was used to find risk factors for AD patients' deaths (Fig. 6). Higher methylation levels on the gene bodies of CAPN3 $(\mathrm{HR}=1.46, \mathrm{CI}=1.19-1.79, P<0.001)$ and STAT5A $(\mathrm{HR}=1.59, \mathrm{CI}=1.29-1.97, P<0.001)$ were associated with significantly increased risk of death in $\mathrm{AD}$ patients, while higher methylation levels on COL2A1 $(\mathrm{HR}=0.80$, $\mathrm{CI}=0.68-0.93, P=0.003), \mathrm{COL} 14 A 1(\mathrm{HR}=0.73, \mathrm{CI}=$ $0.63-0.86, P<0.001)$, and SPOCK3 $(\mathrm{HR}=0.83, \mathrm{CI}=$ $0.70-0.99, P=0.036$ ) were associated with significantly decreased risk of death. Apart from the AD-specific methylome changes, patients have lower death risk if they are younger when first diagnosed with $\mathrm{AD}$ [63]. Peculiarly, male AD patients seem to have greater risk of death [63]. Although BRAAK and CERAD scores are important AD diagnosis criteria, they are barely correlated with AD prognosis. Together, these data suggest that the methylome signatures are more sensitive than traditional clinical markers in determining AD prognosis.

\section{Discussion}

Amyloid deposits and neurofibrillary tangles are wellcharacterized and common pathological features of $\mathrm{AD}$ brains. The aging-related temporal sequence of how these pathological features unfold is well-documented and studied. However, the spatial/regional events of AD 
a

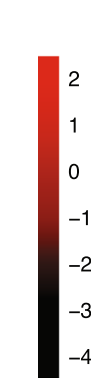

FPKM $\log 2(\mathrm{FC})$

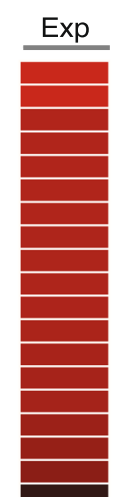

$\underline{-}$
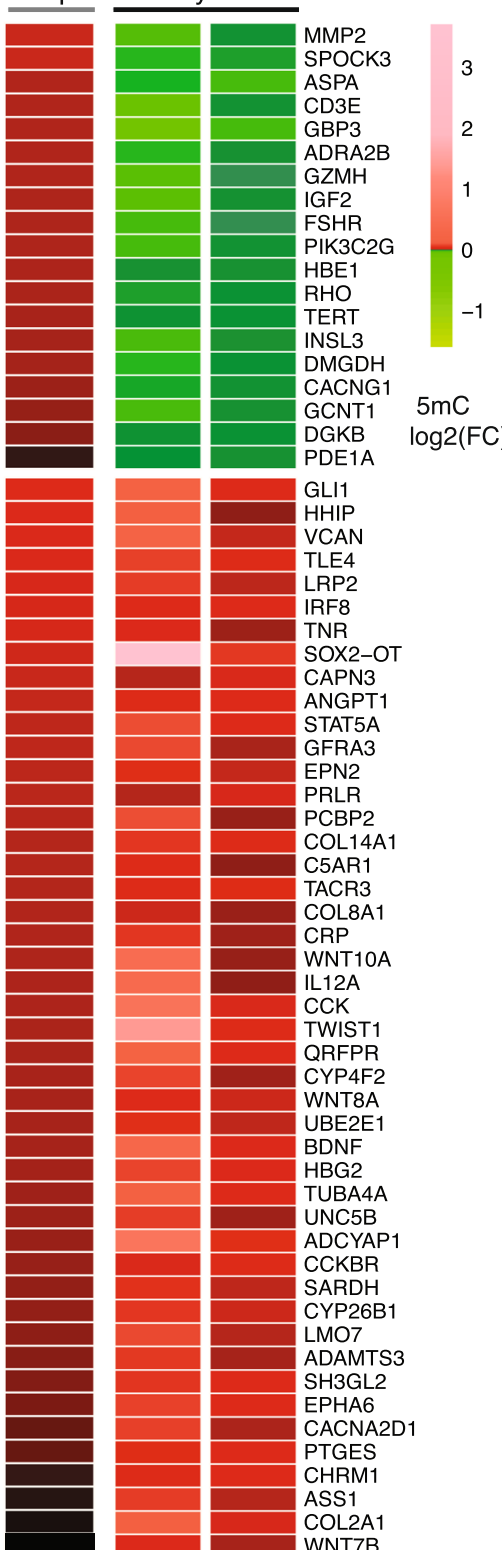

GLI1
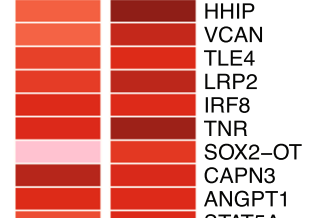

ANGPT1

GFRA3

EPN2

PRL 2

PCBP2

COL14A1

C5AR1

TACR3

COL8A1

COL8A

WNP

IL12A

CCK

TWIST1

QRFPR

QRFPR

WNT8A

UBE2E1

BDNF

BDNF

HBG2

TUBA4A

UNC5B

CCKBR

SARDH

CYP26B1

ADAMTS3

SH3GL2

EPHA6

EPHA6

PACNAS

CHRM

ASS 1

COL2A1

WNT7B

iPSC-derived GSE79144

PSEN2/WT brain

AD/Ctrl b

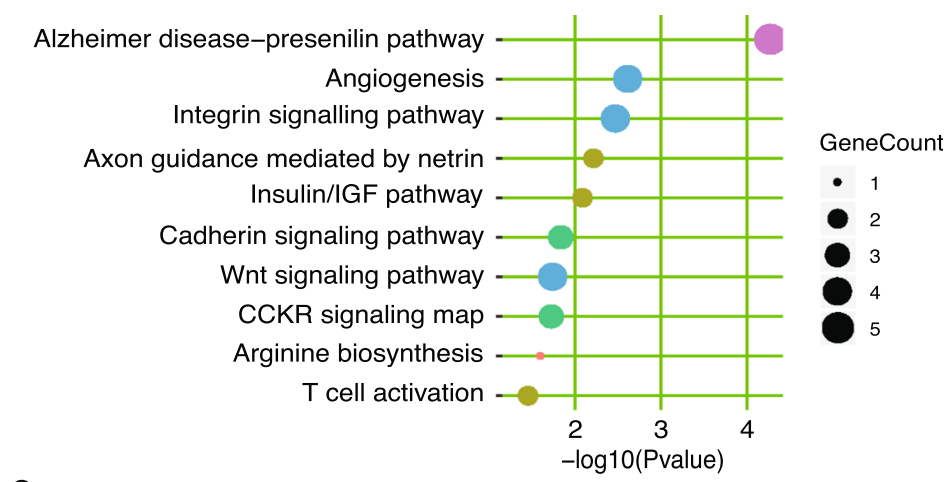

C

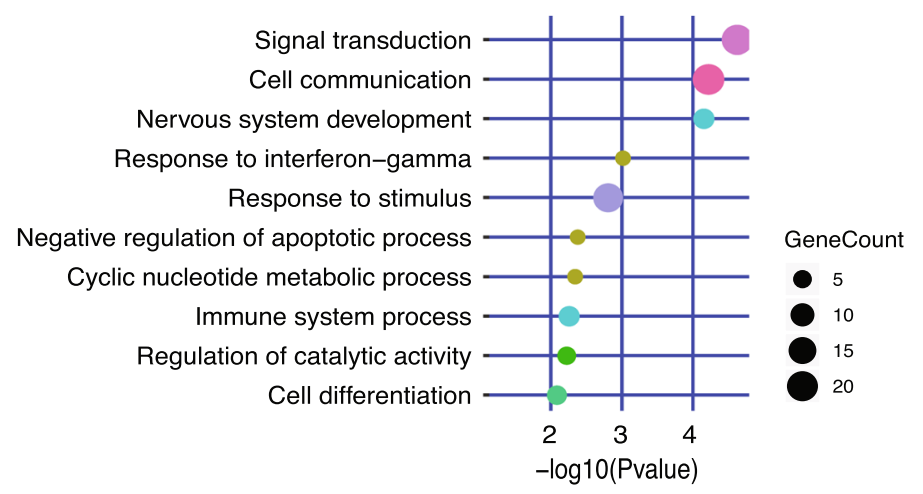

d

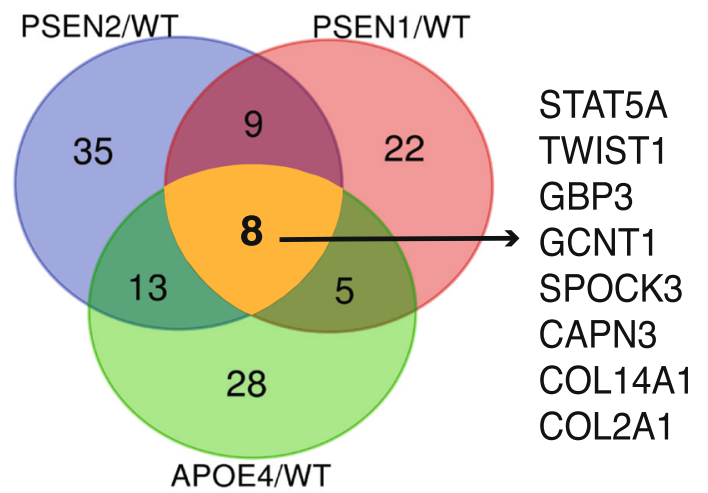

Fig. 5 Cross-validation of key AD genes in independent methylation datasets. a Heatmaps show expression fold-changes and methylation foldchanges in PSEN2NT. The methylation data are from GSE79144 ( $n=44)$. FC, fold-change. b, c PANTHER pathway (b) and GO term (c) enrichment analyses for genes identified from PSEN2NT neurons and validated in the GSE79144 dataset. $\mathbf{d}$ Venn diagrams show the overlap of genes identified from PSEN2/WT, PSEN1/WT, and APOE4/WT neurons and validated in the GSE79144 dataset with a similar methylation change trend

pathogenesis and progression are currently poorly understood, although it has been long noticed that disparities in symptoms and severity of AD progression are specifically associated with neurodegeneration and disordered neurogenesis in different brain regions [64]. Using the experimental strategy outlined in the Graphic Abstract, we are just beginning to understand how different cellular and molecular pathways in different brain regions are linked and contribute to $\mathrm{AD}$ onset and progression. 


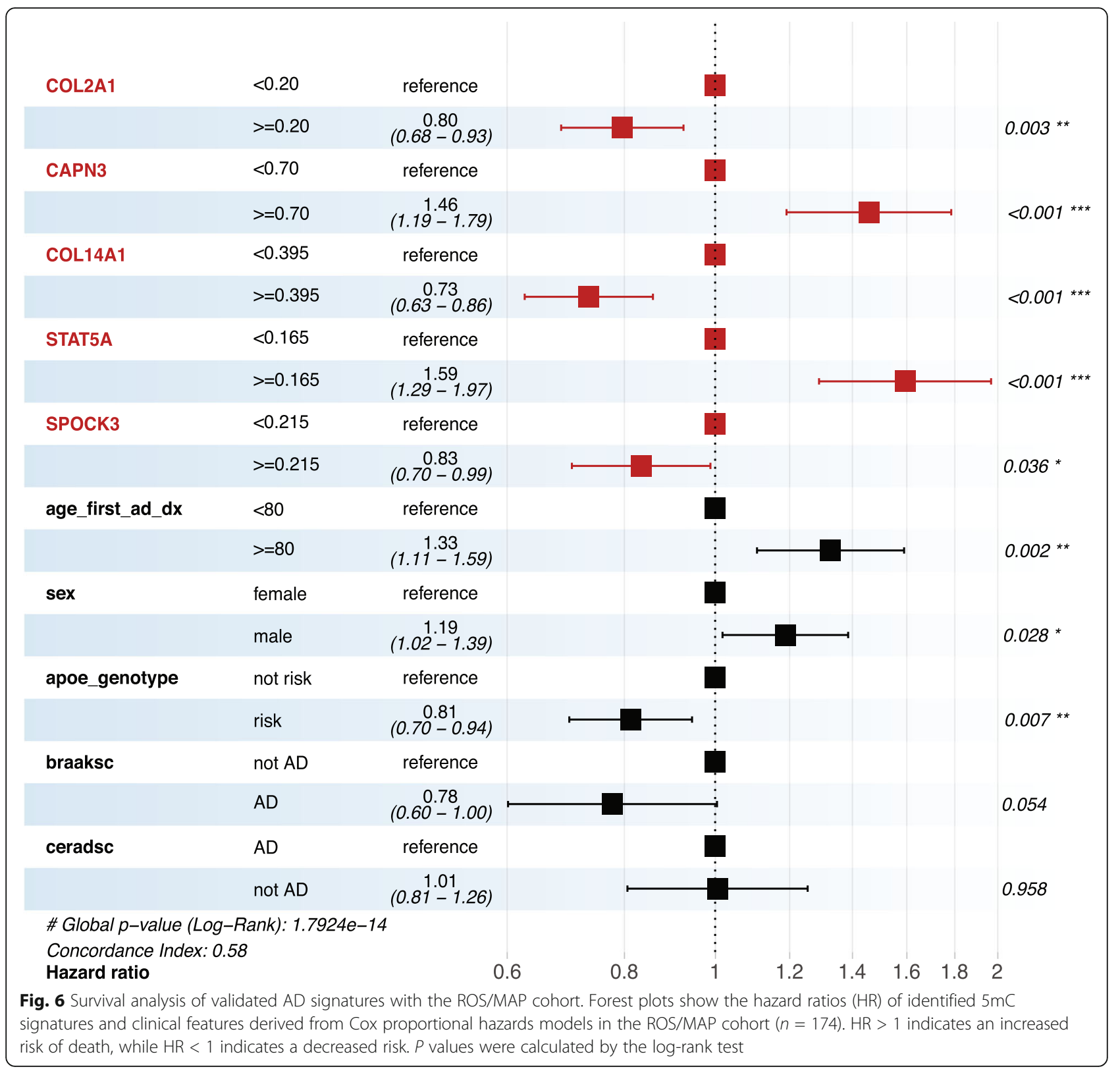

In the present study, we initially analyzed the transcriptomes of 4 dissected brain sub-regions (FUS, HPC, PFC, and TPL) of both AD patients and normal controls. These analyses revealed novel and distinct gene expression signatures in the different brain areas of AD patients. For example, NEUROD1, a well-defined AD risk factor activated by Wnt signaling, which promotes adult neuron maturation [65], was ranked among the top DE genes of the FUS. Concurrently, cytokine-cytokine receptor interaction was the top perturbed pathway in both the HPC and PFC, implying that the immune response is potentially impaired in these areas in AD patients. These findings suggest that there are distinct molecular and cellular mechanisms by which a spatial- specific occurrence of neurodegeneration and impairment of neurogenesis in different brain regions is independently triggered or coordinated to contribute to $\mathrm{AD}$ symptoms.

Selective neuronal loss in vulnerable brain regions is the neuropathological hallmark of AD. Currently, the epigenetic mechanisms of $\mathrm{AD}$ neuronal loss are poorly understood and studied. Functional analysis of the differentially methylated genes in AD showed that a large part of them are associated with neurodevelopment and neurogenesis, indicating that the abnormal DNA methylome in AD may break the normal functional balance in the process of neurodevelopment and neurogenesis, causing potential neuronal loss [66]. By further determining those differentially 
methylated sites and regions, we found that they overlapped with vital regulatory elements in the genome, such as $\mathrm{CpG}$ islands, poised promoters with bivalent histone marks, and enhancers $[19,27,66]$. Enhancer hypomethylation in AD neurons could significantly affect the expression of genes involved in neurogenesis pathways [27]. Poised promoters are essential for neural development and maintenance of lineage differentiation [67]. We speculate that, under the influence of $\mathrm{AD}$, abnormal fluctuations in DNA methylation levels occur on important genomic regulatory elements such as promoters and enhancers, resulting in aberrant expression of genes related to neurodevelopment and neurogenesis. In addition, there are evident epigenetic and transcriptional losses in cell cycle control in AD neurons [27]. For example, hypomethylation of enhancers in AD neurons may upregulate cell cycle genes and promote neuronal death and synapse loss. Therefore, it is possible that hypomethylation of enhancers that affect neurogenesis genes may be the molecular basis and initial cause for a close relationship between the burden of neurofibrillary tangles and neuronal loss in $\mathrm{AD}$ [27].

We discovered that the gene expression signature of $\mathrm{AD}$ in the FUS had the closest similarity to that of in vitro iPSC-derived AD neurons. STRING analysis of the FUS/iPSC-N commonly shared gene expression signature identified a set of genes specifically linked to well-documented key $\mathrm{AD}$ genes such as $A P P$ and $A P O E$. iPSC-derived neurons that resemble the fusiform gyrus in their transcription profiles were generated using a specific and common protocol [34]. Our transcriptional correlation analysis further indicated that the similarity of gene expression signatures between the iPSC-derived neurons and distinct brain regions depends on the differentiation protocols, and possibly the involved growth factors (e.g., NGN2 and GRIN2B). The exact reasons for this are currently unknown and warrant future investigation. The discovery that the iPSC-derived neurons in our study [34] shared a gene expression signature with the FUS provides an excellent and unique tool to study the molecular pathology and mechanisms of action in the FUS during $\mathrm{AD}$ onset and progression. These findings also remind us to re-think the in vitro neuron differentiation process. In particular, extra caution will need to be taken to ensure proper application of a specific neuronal differentiation protocol when establishing models to study neurodegenerative diseases.

We should note that there are several challenges in comparing in vitro data from homogeneous cultures of iPSC-derived neurons with data obtained from highly heterogeneous brain tissues [68-74]. First, the composition of cells in brain tissue is complex. The correlation of expression profiles between FUS and iPSC neurons is likely to be affected by other cell types, such as glia.
Second, when iPSCs are reprogrammed, it is necessary to select appropriate clones to continue the cultivation. This process is not only difficult to standardize, but also merely reflects the nature of a single cell colony. Third, compared with the original neurons of the patient, neurons differentiated in vitro lack the connection and interaction between different brain cells. In future investigations, it is important and necessary to map the cell type- and brain region-specific transcriptional and epigenetic landscapes to disease phenotypes [72]. Nonetheless, despite these potential limitations, it has been reported that transcriptional signatures of schizophrenia in iPSC-derived NPCs and neurons are concordant with post-mortem brains [75]. We are confident that the similarity of gene expression signatures between iPSCneurons and FUS observed in both normal controls and $\mathrm{AD}$ may provide new clues to the molecular mechanisms of AD in FUS.

Genetic and epigenetic risk factors can independently affect the same diseased gene [76]. It is possible that the DNA methylome alterations might be the functional consequence of genetic variants associated with disease susceptibility. On the other hand, epigenetic factors, such as adverse environmental cues or aging, may directly reprogram the epigenome, which, in turn, may alter the expression-associated genes to result in neurodegenerative diseases [76]. In our previous study [17], we reported the first genome-wide roadmap of epigenetic signatures in $\mathrm{AD}$ based on the methylated DNA base cytosine [17]. Significantly, in the present study, we determined novel FUS-specific AD genes, whose transcriptional alterations were significantly linked to key AD risk factors. We identified five AD signature genes with a methylome signature that was significantly associated with AD prognosis in ROS/MAP cohorts. Moreover, the methylome signature is not only restrained to the FUS, but is also commonly shared among other brain regions. We envision that these epigenetic signatures are more generalized and likely to be epigenetic codes for $\mathrm{AD}$ rather than simply the consequence of $\mathrm{AD}$ progression.

\section{Conclusions}

Using a complex of new analytical paradigms that integrates transcriptomes and methylomes of normal controls, AD patients, and "AD-in-dish" models, we identified a set of previously unexplored FUS-specific AD genes (COL2A1, CAPN3, COL14A1, STAT5A, and $S P O C K 3)$ and their epigenetic characteristics, which may provide new insights into the molecular pathology of AD. Moreover, this study first reports the molecular link between FUS and AD, which uncovers the genetic/epigenetic basis of FUS contributing to the spatial/regional events of $\mathrm{AD}$ pathogenesis, leading to new insights into how molecular changes in different brain regions affect 
$\mathrm{AD}$ onset and progression. The FUS-specific genetic/epigenetic signatures may be potential biomarkers for $\mathrm{AD}$ etiology.

\section{Methods \\ Cell lines}

iPSCs and iPSC-derived cortical neurons were obtained from Axol Biosciences (Cambridge, UK). The PSEN1 ${ }^{\text {mut }}$ cell line carries the PSEN1 gene mutation L286V, and the PSEN2 ${ }^{\text {mut }}$ cell line carries the PSEN2 gene mutation N141I. Both types of mutations are genetic risk factors for familial AD. The $\mathrm{APOE}^{\varepsilon 4 / \varepsilon 4}$ cell line carries a homozygote for the APOE $\varepsilon 4$ allele, which is a genetic risk factor for sporadic AD. iPSCs were reprogrammed from skin fibroblasts of $A D$ patients and normal controls. Skin fibroblasts used for reprogramming PSEN1 ${ }^{\text {mut }}$ and PSEN2 ${ }^{\text {mut }}$ cell lines were from 38-year-old female and 81-year-old male AD patients, respectively. Fibroblasts for reprogramming $\mathrm{APOE}^{\varepsilon 4 / \varepsilon 4}$ cell line were from an 87-year-old female patient with sporadic AD. Directed differentiation of iPSCs to cortical neurons was performed as described previously [77].

\section{Oxidative and methylase-assisted bisulfite sequencing}

Oxidative (oxBS-seq) and methylase-assisted bisulfite sequencing (MAB-seq) at single-base-pair resolution were performed as previously described [17]. We performed oxBS-seq and MAB-seq in all cell lines in the AD patient samples reported in this study. All libraries were sequenced using Illumina HiSeq X Ten platform, generating at least $100 \mathrm{~GB}$ of data for each sample, allowing for whole-genome methylation analysis at base resolution.

\section{Identification of $5 \mathrm{mC}, 5 \mathrm{hmC}$, and $5 \mathrm{fC} / 5 \mathrm{caC}$ sites}

We evaluated the data quality of oxBS-seq and MAB-seq [17] and used the bsmap (v2.74) [78] software package to align the reads to the human reference genome of UCSC (hg19) and to identify the methylation signals. We retained CpG sites with a sequencing depth of at least $10 \times$ for downstream analysis. To accurately calculate $5 \mathrm{mC}$ and $5 \mathrm{hmC}$ signals, we used home-made $\mathrm{R}$ script to ensure that the chromosome coordinates of these CpG sites are consistent between each pair of BS and oxBS libraries. Then we used the "mlml" script in methpipe package to identify the $5 \mathrm{mC}$ and $5 \mathrm{hmC}$ signals at these $\mathrm{CpG}$ sites. To calculate $5 \mathrm{fC} / \mathrm{caC}$ signals in the binomial distribution model, we used M.SssI enzyme and bisulfite conversion inefficiency of $1.64 \%$ (measured in preliminary experiments) to correct the $5 \mathrm{fC} / \mathrm{caC}$ signals. We only retained $5 \mathrm{fC} / \mathrm{caC}$ sites with sequencing depth $\geq 10, P$ value $<0.01$, and FDR $<0.01$ for downstream analysis. In addition, given that DNA samples may carry sequence mutations different from the reference genome, this may lead to false positives when calculating the $5 \mathrm{mC}, 5 \mathrm{hmC}$, and $5 \mathrm{fC} / \mathrm{caC}$ signals. We used Biscuit software package (https://github.com/ zwdzwd/biscuit) to identify and remove these potential mutation sites with default parameters. The remaining loci are used for downstream analysis.

\section{Normalization of $5 \mathrm{mC}, 5 \mathrm{hmC}$, and $5 \mathrm{fC} / 5 \mathrm{caC}$ signals}

In order to compare the $5 \mathrm{mC}, 5 \mathrm{hmC}$, and $5 \mathrm{fC} / \mathrm{caC}$ signals between different samples and exclude the effect of the difference in sequencing depth, we scanned the entire genome in non-overlapping $1000 \mathrm{bp}$ bins to normalize the signals. The $5 \mathrm{mC}$ and $5 \mathrm{hmC}$ signals were represented by TNC / (TNC + TNT), where TNT and $\mathrm{TNC}$, respectively, represent the total number of $\mathrm{T}$ and the total number of $\mathrm{C}$ in the region. The $5 \mathrm{fC} / \mathrm{caC}$ signal was represented by the average value in this region, because the density of the $5 \mathrm{fC} / 5 \mathrm{caC}$ sites in the genome is much lower than $5 \mathrm{mC}$ or $5 \mathrm{hmC}$.

\section{mRNA-seq}

The total mRNA was isolated using TRIzol according to the manufacturer's instructions (Invitrogen, CA, USA). Libraries were generated and sequenced at WuxiNextCode (Shanghai, China). For each sample, $>40$ million paired-end reads with Q30 > 90\% were generated. Reads were then mapped onto the hg19 genome using TopHat (v2.1.1) [79]. Quantification of gene expression was performed using featureCounts in the Rsubread package (v1.32.4) [80]. RPKM of genes was calculated using the "rpkm" function in the edgeR package (v3.24.3) [81] and gene annotations were determined through the built-in annotation in the featureCounts package. Differentially expressed genes were identified using the edgeR [81] package with a cutoff RPKM-fold-change $>1.5$ and $P$ value $<0.05$ in $\mathrm{AD}$ samples versus normal controls.

\section{Digital deconvolution of bulk tissues}

Cell-type deconvolution was performed using CIBERSORTx (http://cibersortx.stanford.edu), which is an analytical tool developed by Newman et al. [27] to impute gene expression profiles and provide an estimation of the abundances of member cell types in a mixed cell population, using gene expression data. We used a gene signature matrix (involving 903 cell-specific marker genes) derived from single-cell RNA-seq measures in adult human brain cells (signature matrix [82]; source [83]). CIBERSORTx was run with batch correction and 100 permutations.

\section{Known $A D$ risk factors}

Known AD risk factors were obtained from the MalaCards-HUMAN DISEASE DATABASE (https:// www.malacards.org/card/alzheimer_disease). Susceptible 
genes and risk factors were summarized according to the "Genes" section. The gene list used in our analysis was provided in Table S9.

International Genomics of Alzheimer's Project (IGAP) is a large two-stage study based upon genome-wide association studies (GWAS) on individuals of European ancestry. In stage 1, IGAP used genotyped and imputed data on 7,055,881 single nucleotide polymorphisms (SNPs) to meta-analyze four previously-published GWAS datasets consisting of 17,008 Alzheimer's disease cases and 37,154 controls (The European Alzheimer's disease Initiative (EADI), the Alzheimer Disease Genetics Consortium (ADGC), The Cohorts for Heart and Aging Research in Genomic Epidemiology consortium (CHARGE), The Genetic and Environmental Risk in AD consortium (GERAD)). In stage 2, 11,632 SNPs were genotyped and tested for association in an independent set of 8572 Alzheimer's disease cases and 11,312 controls. Finally, a meta-analysis was performed combining results from stages 1 and 2.

\section{Gene ontology (GO) and Kyoto Encyclopedia of Genes and Genomes (KEGG) enrichment analysis}

GO and KEGG enrichment analyses were performed using the Database for Annotation, Visualization and Integrated Discovery (DAVID) website [84, 85]. Visualization of KEGG pathways was conducted using Pathview (v1.22.3) [86].

\section{Other statistical analyses}

Continuous variables were descriptively summarized using medians with 25th and 75th percentiles, and categorical factors were reported using percentages. $\mathrm{R}$ package "VennDiagram" was used to determine the groupings of values that were presented in the Venn diagram. The Pearson correlation coefficient was calculated to measure the linear correlation of the gene expression between iPSC-derived neurons and the four brain regions $(P$ values $<0.01$ ). The STRING [50] analytical tool was used to reveal the protein-protein interaction (PPI) network, with only high-confidence interaction edges kept for downstream analyses (interaction score $\geq 0.9$, PPI enrichment $P$ value $<1 \mathrm{e}-11)$. Boxplots were used to describe the distribution and patterns of methylation changes in key $\mathrm{AD}$-associated genes. The statistical significance of the methylation changes between different groups was determined by the Wilcoxon rank-sum test using $\mathrm{R}$ package "stats." The multivariable Cox proportional hazards regression model was used to find risk factors for AD patients' deaths. The Kaplan-Meier survival analysis was used to predict the survival probabilities at distinct methylation level cutoffs. $P$ values were calculated by the logrank test.

\section{Supplementary information}

Supplementary information accompanies this paper at https://doi.org/10. 1186/s13148-020-00916-3.

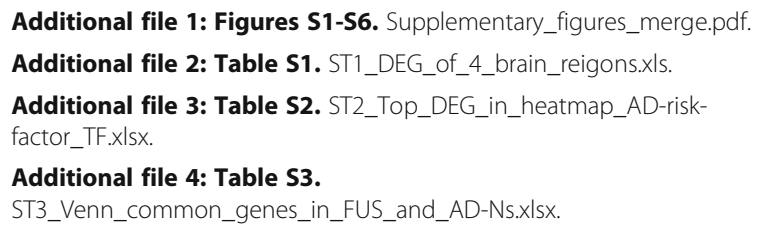

Additional file 1: Figures S1-S6. Supplementary_figures_merge.pdf.

Additional file 2: Table S1. ST1_DEG_of_4_brain_reigons.xls.

Additional file 3: Table S2. ST2_Top_DEG_in_heatmap_AD-riskfactor_TF.xlsx.

Additional file 4: Table S3.

ST3_Venn_common_genes_in_FUS_and_AD-Ns.XIsx.

Additional file 5: Table S4.

ST4_common_up_down_expressed_genes_in_FUS_and_AD-Ns.xlsx.

Additional file 6: Table S5.

ST5_details_of_neurons_differentiation_protocols.xlsx.

Additional file 7: Table S6. ST6_STRING_output_interactions.xlsX. Additional file 8: Table S7.

ST7_validated_genes_function_annotation.xlsx.

Additional file 9: Table S8. ST8_Samples_info.xlsX.

Additional file 10: Table S9. ST9_Known_AD_risk_factors.txt. All computer codes used in our analyses were deposited in GitHub.

\section{Abbreviations}

AD: Alzheimer's disease; FUS: Fusiform gyrus; HPC: Hippocampus; PFC: Prefrontal cortex; TPL: Temporal lobe; DEG: Differentially expressed genes; iPSC: Induced pluripotent stem cell; WT-N, PSEN1-N, PSEN2-N, APOE4$\mathrm{N}$ : iPSC-differentiated neurons, derived from normal individuals and AD patients carrying a PSEN1 mutation, PSEN2 mutation, or homozygous APOE$\varepsilon 4$, respectively; $5 \mathrm{mC}$ : 5 -Methylcytosine; $5 \mathrm{hmC}$ : 5 -Hydroxymethylcytosine; 5fC: 5-Formylcytosine; 5caC: 5-Carboxylcytosine; PPI: Protein-protein interaction; ROS/MAP: The Religious Orders Study and Rush Memory and Aging Project cohorts; EOAD: Early-onset Alzheimer's disease; LOAD: Lateonset Alzheimer's disease

\section{Acknowledgements}

We thank the Rush Alzheimer's Disease Center, Rush University Medical Center, Chicago, for providing access to ROS/MAP study data. We thank A.Prof Frank Gaillard for sharing brain anatomy figures (Radiopaedia.org, rID: 47208, 46670)

We thank the International Genomics of Alzheimer's Project (IGAP) for providing summary results data for these analyses. The investigators within IGAP contributed to the design and implementation of IGAP and/or provided data but did not participate in analysis or writing of this report. IGAP was made possible by the generous participation of the control subjects, the patients, and their families. The i-Select chips was funded by the French National Foundation on Alzheimer's disease and related disorders. EADI was supported by the LABEX (laboratory of excellence program investment for the future) DISTALZ grant, Inserm, Institut Pasteur de Lille, Université de Lille 2, and the Lille University Hospital. GERAD was supported by the Medical Research Council (Grant n 503480), Alzheimer's Research UK (Grant n ${ }^{\circ}$ 503176), the Wellcome Trust (Grant n 082604/2/07/Z), and German Federal Ministry of Education and Research (BMBF):

Competence Network Dementia (CND) grant n $n^{\circ} 01 \mathrm{Gl0102}$, 01G10711, and 01GI0420. CHARGE was partly supported by the NIH/NIA grant R01 AG033193 and the NIA AG081220 and AGES contract N01-AG-12100, the NHLBI grant R01 HL105756, the Icelandic Heart Association, and the Erasmus Medical Center and Erasmus University. ADGC was supported by the NIH/ NIA grants: U01 AG032984, U24 AG021886, U01 AG016976, and the Alzheimer's Association grant ADGC-10-196728.

\section{Authors' contributions}

Y.G.S conceived, designed, and supervised the execution of the entire study. F.W. and D.M. were responsible for bioinformatics analysis and related data acquisition. I.S.F. carried out the oxBS-seq and MAB-seq experiments, and isolated RNA for RNA-seq experiments. H.L. was responsible for the Bioanalyzer experiments. R.F., M.W., J.L. and L.T. have done critical experiments for the original submission. I.I., S.G., W. X., and T.G. worked on critical experimental or analytical data acquisitions for the revisions. Y.G.S., F.W, and D.M. co-wrote 
the manuscript. Y.G.S., I.S.F, D.M., I.I., S. G., F.W., and T.G. edited the manuscript. All authors read and approved the final manuscript.

\section{Authors' information}

About Dr. Yujiang Geno Shi

Dr. Shi discovered the first histone demethylase KDM1/LSD1—which revealed the reversibility of histone methylation and opened up a whole new field in epigenetics (Shi et al., Cell, 2004). He also identified the LSD1 homolog LSD2 and defined the function of the LSD family of histone demethylases in epigenetic gene regulation (Mol. Cell, 2010, 2013, Cell Reports 2019). In addition, he isolated and characterized the first H3K4 trimethylation demethylase SMCX/Jairid1C, directly linking SMCX/Jarid1C mutations with mental retardation and providing insight into the epigenetic mechanisms associated with mental retardation (Tahiliani et al., Nature, 2007). Dr. Shi also studied the ten-eleven translocation (TET) family of 5 methylcytosine $(5 \mathrm{mC})$ dioxygenases involved in DNA demethylation and provided significant insight into the epigenetic mechanisms underlying DNA demethylation and global gene regulation (Xu et al., Cell, 2012). His lab for the first time defined the "loss of $5 \mathrm{hmC}$ " as an epigenetic hallmark of many types of cancers and developed a new method for the comparative and quantitative measurement of both $5 \mathrm{mC}$ and $5 \mathrm{hmC}$ levels and distribution in tumor samples. This work has led to the exploration of $5 \mathrm{hmC}$ as a diagnostic and therapeutic marker for cancer (Lian et al., Cell, 2012). In 2018, Dr. Shi's lab published a Nature paper on the critical discovery of a novel "epigenetic axis" and a "phosphor-switch" in glucose signaling that links diabetes to cancer risk (Di, Nature, 2018). This unprecedented discovery revealed and mechanistically defined an epigenetic base underlying diabetes and cancer development. This Nature work also offered a new molecular mechanism of action of the diabetic drug, metformin, in cancer prevention. In 2019, by using human iPS as a model and the cutting-edge technology of single base $5 \mathrm{mC}$ and $5 \mathrm{hmC}$ sequencing, Dr. Shi's lab discovered the epigenetic signatures of early and late onset Alzheimer's disease (Sci Adv, 2019). These studies emphasize the dynamic interplay of the epigenome and cell signaling, and their importance to Alzheimer's disease.

In addition to the mechanism-oriented basic research, there are multiple translational projects that Dr. Shi's Lab is currently pursuing including (1) deciphering how obesity and diabetes are linked through a new environmental-to-chromatin signaling axis that is able to directly program or reprogram an epigenome, thereby linking these metabolic syndromes to lifethreatening complications; (2) understanding how histone and DNA demethylases regulate the crosstalk between tumors and the immune system and how their inhibition can be integrated into cancer immunotherapy; and (3) characterizing histone modification and DNA methylation aberrancies in neurological disorders/diseases such as in Alzheimer's disease and major depressive disorders (MDD) and dissecting the role of these modifications in disease initiation and progression.

\section{Funding}

This work was partly supported by Biogen Idec Epigenetics consortium grant A220159 for the whole-genome sequencing of $5 \mathrm{mC}, 5 \mathrm{hmC}$, and $5 \mathrm{fC} / \mathrm{caC}$ modifications and RNA sequencing of iPSC-derived neurons.

\section{Availability of data and materials}

Whole-genome sequencing data for $5 \mathrm{mC}$, 5hmC, and $5 \mathrm{fC} / \mathrm{caC}$ modifications have been deposited in the Sequence Read Archive (SRA) with accession code PRJNA476128, along with RNA sequencing data PRJNA557835, and are available from the authors upon request.

All publicly available datasets were already cited when mentioned in the manuscript. The mRNA-seq datasets of different brain regions, including fusiform gyrus (GSE95587), hippocampus (GSE67333), prefrontal cortex (GSE53697), and temporal lobe (GSE104704) (Table S8), were obtained from the GEO website (https://www.ncbi.nlm.nih.gov/geo/). The accession numbers for mRNA-seq data of other iPSC-derived cell lines are GSE87963, GSE90469, GSE107514, GSE111977, GSE112732, GSE114685, GSE115205, GSE102352, GSE102956, GSE114553, GSE58933, GSE63734, and GSE104141 (Table S5).

In order to verify the $A D$ methylation signatures in other independent $A D$ datasets, we analyzed the bisulfite-converted DNA data from 44 brain tissues, including 22 normal controls and 22 AD patients (Table S8) (GSE79144). In addition, in order to assess the potential significance of the AD methylation signatures in the prognosis of $A D$, we performed the analysis of death risks and survival probabilities with the ROS/MAP (Religious Orders Study and Rush Memory and Aging Project) cohort study dataset [62]. The ROS/MAP methylation dataset includes DNA methylation data from 708 subjects' prefrontal cortex tissues.

\section{Ethics approval and consent to participate}

Human data, human tissue, and human participants involved in this study were obtained from public data portals or published studies, which have already received ethics approval and consent from participants.

\section{Consent for publication}

All authors agree to publish this manuscript in Clinical Epigenetics.

\section{Competing interests}

The authors declare that they have no competing interests.

\section{Author details}

'Laboratory of Epigenetics, Institutes of Biomedical Sciences, Fudan University, Shanghai 200032, China. ${ }^{2}$ Key Laboratory of Birth Defects, Children's Hospital of Fudan University, Shanghai 201102, China. ${ }^{3}$ Division of Endocrinology, Diabetes and Hypertension, Department of Medicine, Brigham and Women's Hospital, Harvard Medical School, Boston, MA 02115, USA. ${ }^{4}$ Department of Geriatrics, Shanghai General Hospital, Shanghai 200080, China.

Received: 11 March 2020 Accepted: 10 August 2020

Published online: 27 August 2020

\section{References}

1. Association As. 2018 Alzheimer's disease facts and figures. Alzheimers Dement. 2018;14(3):367-429.

2. Nordberg A. Dementia in 2014. Towards early diagnosis in Alzheimer disease. Nat Rev Neurol. 2015:11(2):69-70.

3. Hampel H, O'Bryant SE, Molinuevo JL, Zetterberg H, Masters CL, Lista S, et al. Blood-based biomarkers for Alzheimer disease: mapping the road to the clinic. Nat Rev Neurol. 2018;14(11):639-52.

4. Sperling RA, Karlawish J, Johnson KA. Preclinical Alzheimer disease-the challenges ahead. Nat Rev Neurol. 2013;9(1):54-8.

5. Tanzi RE. The genetics of Alzheimer disease. Cold Spring Harb Perspect Med. 2012;2(10)

6. Warren JD, Fletcher PD, Golden HL. The paradox of syndromic diversity in Alzheimer disease. Nat Rev Neurol. 2012:8(8):451-64.

7. McKhann GM, Knopman DS, Chertkow H, Hyman BT, Jack CR Jr, Kawas CH, et al. The diagnosis of dementia due to Alzheimer's disease: recommendations from the National Institute on Aging-Alzheimer's Association workgroups on diagnostic guidelines for Alzheimer's disease. Alzheimers Dement. 2011;7(3):263-9.

8. Galton CJ, Patterson K, Xuereb JH, Hodges JR. Atypical and typical presentations of Alzheimer's disease: a clinical, neuropsychological, neuroimaging and pathological study of 13 cases. Brain. 2000;123(Pt 3): 484-98.

9. Kanwisher N, McDermott J, Chun MM. The fusiform face area: a module in human extrastriate cortex specialized for face perception. J Neurosci. 1997; 17(11):4302-11.

10. Bokde AL, Lopez-Bayo P, Meindl T, Pechler S, Born C, Faltraco F, et al. Functional connectivity of the fusiform gyrus during a face-matching task in subjects with mild cognitive impairment. Brain. 2006;129(Pt 5):1113-24.

11. Magistri M, Velmeshev D, Makhmutova M, Faghihi MA. Transcriptomics profiling of Alzheimer's disease reveal neurovascular defects, altered amyloid-beta homeostasis, and deregulated expression of long noncoding RNAs. J Alzheimers Dis. 2015:48(3):647-65.

12. Scheckel C, Drapeau E, Frias MA, Park CY, Fak J, Zucker-Scharff I, et al. Regulatory consequences of neuronal ELAV-like protein binding to coding and non-coding RNAs in human brain. Elife. 2016;5.

13. Nativio R, Donahue G, Berson A, Lan Y, Amlie-Wolf A, Tuzer F, et al. Dysregulation of the epigenetic landscape of normal aging in Alzheimer's disease. Nat Neurosci. 2018;21(4):497-505.

14. Friedman BA, Srinivasan K, Ayalon G, Meilandt WJ, Lin H, Huntley MA, et al. Diverse brain myeloid expression profiles reveal distinct microglial activation states and aspects of Alzheimer's disease not evident in mouse models. Cell Rep. 2018;22(3):832-47. 
15. Isaacson RS, Hristov H, Saif N, Hackett K, Hendrix S, Melendez J, et al. Individualized clinical management of patients at risk for Alzheimer's dementia. Alzheimers Dement. 2019;15(12):1588-602.

16. Wood $\mathrm{H}$. Alzheimer disease: AD-susceptible brain regions exhibit altered DNA methylation. Nat Rev Neurol. 2014;10(10):548.

17. Fetahu IS, Ma D, Rabidou K, Argueta C, Smith M, Liu H, et al. Epigenetic signatures of methylated DNA cytosine in Alzheimer's disease. Sci Adv. 2019;5(8):eaaw2880.

18. Ito S, Shen L, Dai Q, Wu SC, Collins LB, Swenberg JA, et al. Tet proteins can convert 5-methylcytosine to 5-formylcytosine and 5-carboxylcytosine. Science. 2011;333(6047):1300-3.

19. Watson CT, Roussos P, Garg P, Ho DJ, Azam N, Katsel PL, et al. Genomewide DNA methylation profiling in the superior temporal gyrus reveals epigenetic signatures associated with Alzheimer's disease. Genome Med 2016;8(1):5.

20. Sanchez-Mut JV, Aso E, Panayotis N, Lott I, Dierssen M, Rabano A, et al. DNA methylation map of mouse and human brain identifies target genes in Alzheimer's disease. Brain. 2013;136(Pt 10):3018-27.

21. De Jager PL, Srivastava G, Lunnon K, Burgess J, Schalkwyk LC, Yu L, et al. Alzheimer's disease: early alterations in brain DNA methylation at ANK1, BIN1, RHBDF2 and other loci. Nat Neurosci. 2014;17(9):1156-63.

22. Jack CR Jr, Knopman DS, Jagust WJ, Shaw LM, Aisen PS, Weiner MW, et al. Hypothetical model of dynamic biomarkers of the Alzheimer's pathological cascade. Lancet Neurol. 2010;9(1):119-28.

23. Reiman EM, Quiroz YT, Fleisher AS, Chen K, Velez-Pardo C, Jimenez-Del-Rio $M$, et al. Brain imaging and fluid biomarker analysis in young adults at genetic risk for autosomal dominant Alzheimer's disease in the presenilin 1 E280A kindred: a case-control study. Lancet Neurol. 2012;11(12):1048-56.

24. Delgado-Morales R, Esteller M. Opening up the DNA methylome of dementia. Mol Psychiatry. 2017;22(4):485-96.

25. Li P, Marshall L, Oh G, Jakubowski JL, Groot D, He Y, et al. Epigenetic dysregulation of enhancers in neurons is associated with Alzheimer's disease pathology and cognitive symptoms. Nat Commun. 2019;10(1):2246.

26. Lardenoije R, Roubroeks JAY, Pishva E, Leber M, Wagner $H$, latrou A, et al. Alzheimer's disease-associated (hydroxy)methylomic changes in the brain and blood. Clin Epigenetics. 2019;11(1):164.

27. Newman AM, Steen CB, Liu CL, Gentles AJ, Chaudhuri AA, Scherer F, et al. Determining cell type abundance and expression from bulk tissues with digital cytometry. Nat Biotechnol. 2019;37(7):773-82.

28. Lucas JJ, Hernandez F, Gomez-Ramos P, Moran MA, Hen R, Avila J. Decreased nuclear beta-catenin, tau hyperphosphorylation and neurodegeneration in GSK-3beta conditional transgenic mice. EMBO J. 2001; 20(1-2):27-39.

29. Peineau S, Taghibiglou C, Bradley C, Wong TP, Liu L, Lu J, et al. LTP inhibits LTD in the hippocampus via regulation of GSK3beta. Neuron. 2007;53(5):703-17.

30. Rice HC, de Malmazet D, Schreurs A, Frere S, Van Molle I, Volkov AN, et al. Secreted amyloid-beta precursor protein functions as a GABABR1a ligand to modulate synaptic transmission. Science. 2019;363:6423.

31. Lambert JC, Ibrahim-Verbaas CA, Harold D, Naj AC, Sims R, Bellenguez C, et al. Meta-analysis of 74,046 individuals identifies 11 new susceptibility loci for Alzheimer's disease. Nat Genet. 2013:45(12):1452-8.

32. Bertram L, McQueen MB, Mullin K, Blacker D, Tanzi RE. Systematic metaanalyses of Alzheimer disease genetic association studies: the AlzGene database. Nat Genet. 2007;39(1):17-23.

33. LaFerla FM, Green KN. Animal models of Alzheimer disease. Cold Spring Harb Perspect Med. 2012;2:11.

34. Shi Y, Kirwan P, Livesey FJ. Directed differentiation of human pluripotent stem cells to cerebral cortex neurons and neural networks. Nat Protoc. 2012; 7(10):1836-46.

35. Busskamp V, Lewis NE, Guye P, Ng AH, Shipman SL, Byrne SM, et al. Rapid neurogenesis through transcriptional activation in human stem cells. Mol Syst Biol. 2014;10:760.

36. Chen $C$, Jiang $P$, Xue H, Peterson SE, Tran HT, McCann AE, et al. Role of astroglia in Down's syndrome revealed by patient-derived human-induced pluripotent stem cells. Nat Commun. 2014;5:4430.

37. Hammond TR, Stevens B. Increasing the neurological-disease toolbox using iPSC-derived microglia. Nat Med. 2016;22(11):1206-7.

38. Zhang Y, Pak C, Han Y, Ahlenius H, Zhang Z, Chanda S, et al. Rapid singlestep induction of functional neurons from human pluripotent stem cells. Neuron. 2013;78(5):785-98.
39. Dell'Anno MT, Wang X, Onorati M, Li M, Talpo F, Sekine $Y$, et al. Human neuroepithelial stem cell regional specificity enables spinal cord repair through a relay circuit. Nat Commun. 2018;9(1):3419.

40. Sarkar A, Mei A, Paquola ACM, Stern S, Bardy C, Klug JR, et al. Efficient generation of CA3 neurons from human pluripotent stem cells enables modeling of hippocampal connectivity in vitro. Cell Stem Cell. 2018;22(5): 684-97 e9.

41. Swarup V, Hinz Fl, Rexach JE, Noguchi Kl, Toyoshiba H, Oda A, et al. Identification of evolutionarily conserved gene networks mediating neurodegenerative dementia. Nat Med. 2019;25(1):152-64.

42. Kriks S, Shim JW, Piao J, Ganat YM, Wakeman DR, Xie Z, et al. Dopamine neurons derived from human ES cells efficiently engraft in animal models of Parkinson's disease. Nature. 2011:480(7378):547-51.

43. Nehme R, Zuccaro E, Ghosh SD, Li C, Sherwood JL, Pietilainen O, et al. Combining NGN2 programming with developmental patterning generates human excitatory neurons with NMDAR-mediated synaptic transmission. Cell Rep. 2018;23(8):2509-23.

44. Bell $S$, Maussion $G$, Jefri M, Peng $H$, Theroux JF, Silveira $H$, et al. Disruption of GRIN2B impairs differentiation in human neurons. Stem Cell Reports. 2018; $11(1): 183-96$

45. Yuan F, Chen X, Fang KH, Wang Y, Lin M, Xu SB, et al. Induction of human somatostatin and parvalbumin neurons by expressing a single transcription factor LIM homeobox 6. Elife. 2018;7.

46. Yu DX, Di Giorgio FP, Yao J, Marchetto MC, Brennand K, Wright R, et al. Modeling hippocampal neurogenesis using human pluripotent stem cells. Stem Cell Reports. 2014;2(3):295-310.

47. Brennand KJ, Simone A, Jou J, Gelboin-Burkhart C, Tran N, Sangar S, et al. Modelling schizophrenia using human induced pluripotent stem cells. Nature. 2011;473(7346):221-5.

48. Bajpai R, Chen DA, Rada-Iglesias A, Zhang J, Xiong Y, Helms J, et al. CHD7 cooperates with PBAF to control multipotent neural crest formation. Nature. 2010;463(7283):958-62

49. Huang EJ, Reichardt LF. Neurotrophins: roles in neuronal development and function. Annu Rev Neurosci. 2001:24:677-736.

50. Szklarczyk D, Franceschini A, Wyder S, Forslund K, Heller D, Huerta-Cepas J, et al. STRING v10: protein-protein interaction networks, integrated over the tree of life. Nucleic Acids Res. 2015;43(Database issue):D447-52.

51. Corder EH, Saunders AM, Strittmatter WJ, Schmechel DE, Gaskell PC, Small GW, et al. Gene dose of apolipoprotein E type 4 allele and the risk of Alzheimer's disease in late onset families. Science. 1993:261(5123):921-3.

52. Wang LL, Pan XL, Wang Y, Tang HD, Deng YL, Ren RJ, et al. A single nucleotide polymorphism in LRP2 is associated with susceptibility to Alzheimer's disease in the Chinese population. Clin Chim Acta. 2011;412(34):268-70.

53. Kundra R, Ciryam P, Morimoto RI, Dobson CM, Vendruscolo M. Protein homeostasis of a metastable subproteome associated with Alzheimer's disease. Proc Natl Acad Sci U S A. 2017;114(28):E5703-E11.

54. Hokama M, Oka S, Leon J, Ninomiya T, Honda H, Sasaki K, et al. Altered expression of diabetes-related genes in Alzheimer's disease brains: the Hisayama study. Cereb Cortex. 2014;24(9):2476-88.

55. Thauvin-Robinet C, Auclair M, Duplomb L, Caron-Debarle M, Avila M, StOnge J, et al. PIK3R1 mutations cause syndromic insulin resistance with lipoatrophy. Am J Hum Genet. 2013:93(1):141-9.

56. Hancock ML, Meyer RC, Mistry M, Khetani RS, Wagschal A, Shin T, et al. Insulin receptor associates with promoters genome-wide and regulates gene expression. Cell. 2019;177(3):722-36 e22.

57. Iwai $T$, linuma $Y$, Kodani $R$, Oka J. Neuromedin U inhibits inflammationmediated memory impairment and neuronal cell-death in rodents. Neurosci Res. 2008:61(1):113-9.

58. Do C, Lang CF, Lin J, Darbary H, Krupska I, Gaba A, et al. Mechanisms and disease associations of haplotype-dependent allele-specific DNA methylation. Am J Hum Genet. 2016;98(5):934-55.

59. Natarajan C, Sriram S, Muthian G, Bright JJ. Signaling through JAK2-STAT5 pathway is essential for IL-3-induced activation of microglia. Glia. 2004;45(2): 188-96.

60. Leidinger P, Backes C, Deutscher S, Schmitt K, Mueller SC, Frese K, et al. A blood based 12-miRNA signature of Alzheimer disease patients. Genome Biol. 2013:14(7):R78.

61. Chen D, Yang $X$, Yang J, Lai G, Yong T, Tang $X$, et al. Prebiotic effect of fructooligosaccharides from Morinda officinalis on Alzheimer's disease in 
rodent models by targeting the microbiota-gut-brain axis. Front Aging Neurosci. 2017;9:403.

62. Bennett DA, Buchman AS, Boyle PA, Barnes LL, Wilson RS, Schneider JA. Religious orders study and rush memory and aging project. J Alzheimers Dis. 2018;64(s1):S161-S89.

63. Zanetti O, Solerte SB, Cantoni F. Life expectancy in Alzheimer's disease (AD). Arch Gerontol Geriatr. 2009;49(Suppl 1):237-43.

64. Liu CC, Liu CC, Kanekiyo T, Xu H, Bu G. Apolipoprotein E and Alzheimer disease: risk, mechanisms and therapy. Nat Rev Neurol. 2013;9(2):106-18.

65. Gao Z, Ure K, Ables JL, Lagace DC, Nave KA, Goebbels S, et al. Neurod1 is essential for the survival and maturation of adult-born neurons. Nat Neurosci. 2009;12(9):1090-2.

66. Altuna M, Urdanoz-Casado A, Sanchez-Ruiz de Gordoa J, Zelaya MV Labarga A, Lepesant JMJ, et al. DNA methylation signature of human hippocampus in Alzheimer's disease is linked to neurogenesis. Clin Epigenetics. 2019;11(1):91.

67. Maupetit-Mehouas S, Montibus B, Nury D, Tayama C, Wassef M, Kota SK et al. Imprinting control regions (ICRs) are marked by mono-allelic bivalent chromatin when transcriptionally inactive. Nucleic Acids Res. 2016;44(2):621-35.

68. Gasparoni G, Bultmann S, Lutsik P, Kraus TFJ, Sordon S, Vlcek J, et al. DNA methylation analysis on purified neurons and glia dissects age and Alzheimer's disease-specific changes in the human cortex. Epigenetics Chromatin. 2018;11(1):41.

69. Iwamoto K, Bundo M, Ueda J, Oldham MC, Ukai W, Hashimoto E, et al. Neurons show distinctive DNA methylation profile and higher interindividual variations compared with non-neurons. Genome Res. 2011; 21(5):688-96.

70. Lake BB, Ai R, Kaeser GE, Salathia NS, Yung YC, Liu R, et al. Neuronal subtypes and diversity revealed by single-nucleus RNA sequencing of the human brain. Science. 2016;352(6293):1586-90.

71. Negi SK, Guda C. Global gene expression profiling of healthy human brain and its application in studying neurological disorders. Sci Rep. 2017;7(1):897.

72. Rizzardi LF, Hickey PF, Rodriguez DiBlasi V, Tryggvadottir R, Callahan CM, Idrizi A, et al. Neuronal brain-region-specific DNA methylation and chromatin accessibility are associated with neuropsychiatric trait heritability. Nat Neurosci. 2019;22(2):307-16.

73. Tasic B, Menon V, Nguyen TN, Kim TK, Jarsky T, Yao Z, et al. Adult mouse cortical cell taxonomy revealed by single cell transcriptomics. Nat Neurosci. 2016:19(2):335-46.

74. Zeisel A, Munoz-Manchado AB, Codeluppi S, Lonnerberg P, La Manno G, Jureus $A$, et al. Brain structure. Cell types in the mouse cortex and hippocampus revealed by single-cell RNA-seq. Science. 2015;347(6226): $1138-42$.

75. Hoffman GE, Hartley BJ, Flaherty E, Ladran I, Gochman P, Ruderfer DM, et al. Transcriptional signatures of schizophrenia in hiPSC-derived NPCs and neurons are concordant with post-mortem adult brains. Nat Commun 2017:8(1):2225

76. Klein HU, De Jager PL. Uncovering the role of the methylome in dementia and neurodegeneration. Trends Mol Med. 2016;22(8):687-700.

77. Shi Y, Kirwan P, Smith J, Robinson HP, Livesey FJ. Human cerebral cortex development from pluripotent stem cells to functional excitatory synapses. Nat Neurosci. 2012;15(3):477-86 S1.

78. Xi Y, Li W. BSMAP: whole genome bisulfite sequence MAPping program. BMC Bioinformatics. 2009:10:232.

79. Trapnell C, Pachter L, Salzberg SL. TopHat: discovering splice junctions with RNA-Seq. Bioinformatics. 2009;25(9):1105-11.

80. Liao Y, Smyth GK, Shi W. The R package Rsubread is easier, faster, cheaper and better for alignment and quantification of RNA sequencing reads. Nucleic Acids Res. 2019;47(8):e47.

81. Robinson MD, McCarthy DJ, Smyth GK. edgeR: a Bioconductor package for differential expression analysis of digital gene expression data. Bioinformatics. 2010;26(1):139-40.

82. Yu Q, He Z. Comprehensive investigation of temporal and autism-associated cell type composition-dependent and independent gene expression changes in human brains. Sci Rep. 2017;7(1):4121.

83. Darmanis S, Sloan SA, Zhang Y, Enge M, Caneda C, Shuer LM, et al. A survey of human brain transcriptome diversity at the single cell level. Proc Natl Acad Sci U S A. 2015;112(23):7285-90.
84. Huang da W, Sherman BT, Lempicki RA. Systematic and integrative analysis of large gene lists using DAVID bioinformatics resources. Nat Protoc. 2009; 4(1):44-57.

85. Huang da W, Sherman BT, Lempicki RA. Bioinformatics enrichment tools: paths toward the comprehensive functional analysis of large gene lists. Nucleic Acids Res. 2009;37(1):1-13.

86. Luo W, Pant G, Bhavnasi YK, Blanchard SG Jr, Brouwer C. Pathview Web: user friendly pathway visualization and data integration. Nucleic Acids Res. 2017:45(W1):W501-W8

\section{Publisher's Note}

Springer Nature remains neutral with regard to jurisdictional claims in published maps and institutional affiliations.
Ready to submit your research? Choose BMC and benefit from:

- fast, convenient online submission

- thorough peer review by experienced researchers in your field

- rapid publication on acceptance

- support for research data, including large and complex data types

- gold Open Access which fosters wider collaboration and increased citations

- maximum visibility for your research: over $100 \mathrm{M}$ website views per year

At BMC, research is always in progress.

Learn more biomedcentral.com/submissions 